\title{
Psicoterapia Psicodinâmica e Tratamento Biológico com Fluoxetina: Comparação de Resposta Cognitiva em Pacientes Deprimidos ${ }^{1}$
}

\author{
Andre Goettems Bastos ${ }^{2}$ \\ Clarissa Marceli Trentini \\ Universidade Federal do Rio Grande do Sul
}

\begin{abstract}
RESUMO - A depressão é uma doença grave, com repercussões importantes no humor e na cognição. Tratamentos farmacológicos e/ou psicoterápicos estão comumente indicados. O presente estudo objetivou avaliar e comparar a cognição de pacientes deprimidos antes e após 12 meses de tratamento com fluoxetina ou psicoterapia psicodinâmica. Cento e oitenta pacientes foram divididos em dois grupos, e avaliados por meio da WAIS-III. Os resultados mostraram uma melhora significativa em diferentes subtestes da WAIS-III. A MANOVA indicou que há uma diferença significativa entre os grupos nas pontuações médias obtidas na reavaliação 12 meses após o início dos tratamentos. Os resultados sugerem que a psicoterapia psicodinâmica e a terapia com fluoxetina agem de forma diferente na cognição de pacientes deprimidos.
\end{abstract}

Palavras-chave: psicoterapia psicodinâmica, fluoxetina, cognição, depressão

\section{Psychodynamic Psychotherapy and Biological Treatment with Fluoxetine: Comparison of Cognitive Response in Depressed Patients}

\begin{abstract}
Depression is a serious illness with an important impact on humor and cognition. Pharmacological treatments and/or psychotherapy are commonly indicated. The present study aimed to compare cognition in adult patients diagnosed with depression before and after 12 months of treatment with fluoxetine or psychodynamic psychotherapy. One hundred and eighty depressed patients were divided into two treatment groups, and cognitively assessed with the WAIS-III. Results showed a significant improvement in different subtests of WAIS-III. MANOVA indicated that there is a significant difference between groups in the mean scores of the post-treatment assessment. The results suggest that psychodynamic psychotherapy and fluoxetine act differently on cognition of depressed patients.
\end{abstract}

Keywords: psychodynamic psychotherapy, fluoxetine, cognition, depression

Desde o final do século XX, alguns pesquisadores têm procurado, no contexto da psicoterapia psicodinâmica (ou psicanalítica), evidências de alterações reais no funcionamento da pessoa, tanto no que se refere às questões psicodinâmicas, como às questões cognitivas. Destaca-se aqui o trabalho de Andreassen (1997) no reconhecimento de que a mente é uma atividade do cérebro e de sua cognição, e que estão intrinsecamente ligados.

Gabbard $(2000,2008)$ salienta que a psicoterapia tem um impacto sobre o cérebro - via mente. Isso é corroborado por diversos autores, como Fonagy, Roth e Higgitt (2005), Kandel (2003) e Solms e Turnbull (2002). Da mesma forma, as alterações neurológicas e cognitivas, ou seus efeitos sobre o sujeito, constituem uma ampla área de estudos (Hyman, 1999; Kaplan-Solms \& Solms, 2005; Robinson, 2004).

O presente estudo objetiva analisar e comparar mudanças cognitivas em pacientes deprimidos ao longo de dois modelos de tratamento, a saber: tratamento com psicoterapia psicodinâmica e tratamento biológico com fluoxetina, durante o período de 12 meses. As funções avaliadas incluem atenção, memória imediata, amplitude da informação, capacidade de julgamento, compreensão lógica e verbal, nível de formação

1 Apoio: CNPq.

2 Endereço para correspondência: Rua Alfredo Miranda Obino 240, Porto Alegre, RS, Brasil, CEP: 91225-100.E-mail: andregbastos@gmail.com de conceitos, pensamento lógico, nível da capacidade de abstração, capacidade de análise e síntese, concentração, habilidade matemática, memória auditiva, capacidade de aprendizagem, memória visual, percepção de detalhes, organização visual, capacidade de antecipação e interpretação de situações sociais, capacidade de metodização, rapidez de sintetização e raciocínio.

Essas diferentes funções e seu desempenho em processos depressivos foram examinados na literatura (Chepenik, Cornew, \& Farah, 2007; Dunkin et al., 2000; Harvey et al., 2004; Marvel \& Paradiso, 2004; Murphy, Nimmo-Smith, \& Lawrence, 2003; Rogers et al., 2004). Alterações no funcionamento cognitivo de pacientes deprimidos são corroboradas por estudos de neuroimagem que indicam alterações no funcionamento cerebral em pacientes deprimidos (Eugene et al., 2003; Rogers et al., 2004; Steele \& Lawrie, 2004).

A capacidade de utilizar a cognição em uma potencialidade saudável, por meio de pensamentos funcionais, e conduta adaptável, monitorando também os afetos, constitui uma das propriedades nucleares dos modelos contemporâneos de psicoterapia psicodinâmica (Fonagy, Roth, \& Higgit, 2005; Gabbard, 2004; Lamb, 2005; Leichsenring \& Rabung, 2008). No entanto, o tema é praticamente desconsiderado na literatura da área. Geralmente, associa-se a psicoterapia cognitivo-comportamental a mudanças cognitivas em pacientes (Dobson, 1989; Vittengl, Clark, Dunn, \& Jarrett, 
2007). Apesar da ampla busca efetuada no PubMed, PsycInfo, MedLine, Scopus e ScienceDirect utilizando-se de descritores que associavam os termos psychodynamic, psychoanalytic, psychoanalysis AND cognition AND cognitive e assemelhados (por exemplo, neuropsychological), foram encontrados alguns poucos artigos relacionando alterações cognitivas com o tratamento psicoterápico psicodinamicamente orientado. Yazigi et al. (2011), por exemplo, relataram alterações cognitivas em pacientes recebendo psicoterapia psicanalítica de longo prazo. Essas alterações estavam associadas, principalmente, a um aumento na capacidade de atenção e na velocidade de processamento cognitivo. No entanto, estudos visando encontrar alterações cognitivas no contexto de tratamentos dinamicamente orientados podem ser considerados raros.

A psicoterapia psicodinâmica é um método de tratamento proveniente da psicanálise idealizado no começo do século XX por Sigmund Freud. O próprio termo psicodinâmica, em geral, se refere a uma abordagem que tem sua origem na teoria e no conhecimento psicanalíticos (Kaplan \& Sadock, 2008).

Freud (1905/1996, 1918/1996, 1933/1996, 1937/1996) citou a variedade de formas de psicoterapias existentes na relação terapeuta-paciente, colocando a necessidade de melhor estudá-las e sistematizá-las. A técnica de Freud foi denominada por ele mesmo de "psicoterapia psicanalítica" (1905, p. 242), e a partir dessa origem, foram-se criando modelos alternativos que respeitam os princípios técnicos e teóricos da psicoterapia psicanalítica. As principais diferenças nos princípios técnicos parecem ser as menores frequências de consultas semanais (uma ou duas, ao invés de, no mínimo, três na psicanálise), a "abolição" do divã em favorecimento ao encontro face-a-face (face-to-face) entre paciente e terapeuta, e intervenções mais integradoras (Machado \& Vasconcellos, 1998).

Em relação aos resultados esperados da psicoterapia psicodinâmica, alguns autores (Bachrach, Galatzer-Levy, Skolnikoff, \& Waldron, 1991; Greene, 1993; Wallerstein, 1986) colocam que é "observada" uma diminuição da angústia, um aumento na capacidade de racionalização dos conflitos, da tolerância à frustração, da capacidade de introspecção e de antecipação da detecção de possíveis estressores ambientais. Além disso, há inúmeros relatos, em estudos de caso, a respeito de alterações na forma de perceber, interpretar e se comportar em relação ao mundo e às outras pessoas.

Da mesma forma, pesquisas empíricas em psicoterapia psicodinâmica (Leuzinger-Bohleber, Stuhr, Bernhard, \& Manfred, 2003; Roth \& Fonagy, 2005) têm apresentado resultados no que tange a alterações no funcionamento global dos pacientes (incluindo afeto, comportamento e cognição), e consequente efetividade do tratamento. $\mathrm{O}$ termo "cognição" abrange funções neuropsicológicas como atenção, sensopercepção, memória, orientação, consciência, pensamento, linguagem, inteligência, afeto e conduta, entre outras. Algumas dessas funções podem estar comprometidas em distúrbios neuropsiquiátricos. Assim, por meio da avaliação e reavaliação da cognição de tempos em tempos, pode ser possível acompanhar o próprio andamento do processo psicoterápico, no sentido de saber se a cognição está “evoluindo" nos potenciais que a doença psíquica notadamente costuma atacar em determinadas patologias (Porter, Bourke, \& Gallagher, 2007).

\section{Transtorno depressivo maior}

No presente estudo, a patologia em questão é a depressão (conforme DSM-IV-TR, APA, 2002), a qual apresenta a maior incidência na estatística das doenças mentais (World Health Organization - WHO, 2009). Segundo a WHO, a depressão é uma doença mental comum, que costumeiramente é caracterizada por tristeza, perda de interesse e motivação para atividades e falta de energia para as tarefas do cotidiano. É estimado que $5-10 \%$ da população de todas as idades esteja sofrendo de depressão identificável que necessite de tratamento. O risco de desenvolver depressão ao longo do curso de vida é de $10-20 \%$ em mulheres e um pouco menor em homens.

Apesar da seriedade da depressão como uma doença e da disponibilidade de tratamentos reconhecidamente eficazes, apenas 30\% dos casos em todo o mundo recebem os cuidados necessários (WHO, 2009). Os transtornos depressivos são comuns, causando um nível muito alto de sofrimento. As estimativas da OMS mostram que ao redor do ano de 2020 a depressão será a segunda doença, entre todas as existentes, mais presente em todas as faixas etárias, em ambos os sexos, em todos os países do mundo. Além disso, ela é causa de incapacitação funcional e comportamental do sujeito, pois atinge aspectos motivacionais da vida, abalando a personalidade e afetando a cognição (Zorzetto Filho, 2009).

\section{Aspectos cognitivos na depressão}

Alterações cognitivas geralmente estão presentes em pacientes deprimidos (Chepenik, Cornew, \& Farah, 2007). A literatura demonstra principalmente alterações no processamento e organização do conteúdo perceptual, na memória operacional, na capacidade de atenção, nas funções executivas, no controle cognitivo e nos processos inibitórios, e na velocidade de processamento cognitivo (Harvey et al., 2004; Marvel \& Paradiso, 2004; Philips, Drevets, Rauch \& Lane, 2003a, 2003b; Rogers et al., 2004; Steele \& Lawrie, 2004). O DSM-IV-TR (2002) inclui, entre os sintomas apresentados na depressão, retardo psicomotor e concentração diminuída.

$\mathrm{Na}$ verdade, costuma-se associar somente as depressões mais graves com comprometimentos nos desempenhos de testes neuropsicológicos (Robbins, Joyce \& Sahakian, 1992; Zorzetto Filho, 2009). No entanto, Miller (1975) já havia expandido essa ideia, evidenciando a associação de formas moderadas de depressão a comprometimentos em várias tarefas cognitivas, motoras, perceptivas e de comunicação. Esse autor localizou déficits cognitivos na inteligência global, na capacidade visoespacial, na memória e na aprendizagem, na capacidade de raciocínio abstrato, na linguagem e na velocidade de processamento. Sua conclusão foi a de que em todos os tipos de depressão existe algum nível de prejuízo cognitivo.

De modo geral, muitos estudos demonstram que a depressão exerce uma ampla e consistente influência sobre vários aspectos da cognição. Isso levanta a possibilidade de 
que a cognição como um todo acaba por ser atacada pela instalação da depressão. Os achados de que uma melhora cognitiva ocorre antes da melhora do humor do deprimido durante o tratamento traz sustentação a essa possibilidade (Dunkin et al., 2000).

Além disso, os achados neuropsicológicos de alterações na cognição de pacientes deprimidos são corroborados por achados de neuroimagem (Drevets, 2003; Marvel \& Paradiso, 2004). Aparentemente, em pacientes deprimidos, há uma variada alteração da neuroanatomia funcional (Murphy et al., 2003).

\section{Tratamentos para depressão}

Como visto anteriormente, um dos tratamentos para depressão é a psicoterapia psicodinâmica. No entanto, os tratamentos para depressão incluem diversas formas de psicoterapias e/ou medicações antidepressivas (Kaplan \& Sadock, 2008). O tipo de tratamento mais indicado ainda é motivo de estudos (Greenberg \& Goldman, 2009; Tomba \& Fava, 2009), e entre os tratamentos, estão as psicoterapias, o uso de psicofármacos e o tratamento combinado com ambos (Greenberg \& Goldberg, 2009; Tomba \& Fava, 2009).

Segundo Cuijpers, Clignet, Meijel, Straten e Andersson (2011), as psicoterapias mais utilizadas para o tratamento da depressão em adultos são as de enfoque cognitivo-comportamental, a interpessoal e a psicanalítica (ou psicodinâmica). Ao avaliar os tratamentos psicoterápicos para depressão, Lambert e Ogles (2004) fizeram uma revisão de 19 metanálises de estudos publicados entre 1983 e 2001, e descobriram que os resultados das psicoterapias eram positivos. Isso foi corroborado pelo estudo de Westen e Morrison (2001), que demonstrou ampla melhora nos pacientes em diferentes formas de psicoterapia.

Desde a década de 1950, com a descoberta da ação terapêutica dos antidepressivos tricíclicos (ADTs) e dos inibidores da monoaminoxidase (IMAOs), diversos grupos de fármacos foram acrescentados ao arsenal terapêutico, tais como os inibidores seletivos da recaptação da serotonina (ISRSs), os inibidores da recaptação de serotonina e noradrenalina (IRSNs), o antagonista noradrenérgico e serotoninérgico específico (mirtazapina), os antagonistas 5-HT2, a bupropiona e, mais recentemente, a agomelatina (Sena, 2009). Nenhum antidepressivo influencia de forma acentuada o organismo normal, apenas corrigem condições de funcionamento anômalo (Moreno, Moreno, \& Soares, 2002).

Entre os antidepressivos, os inibidores da recaptação da serotonina (ISRSs) constituem-se em medicamentos eficazes, com poucos problemas de tolerabilidade e segurança (Kaplan \& Sadock, 2008), sendo considerados medicamentos de primeira linha (Tolman, 2009). E entre os ISRSs, a fluoxetina tornou-se um dos mais populares do mundo por meio do Prozac ${ }^{\circ}$.

A dose típica de fluoxetina é de 20 a $60 \mathrm{mg}$ por dia (Tolman, 2009). A fluoxetina é bem absorvida após administração oral, e seu pico de concentração plasmática ocorre seis a sete horas após a ingestão da dose inicial (Sena, 2009). A meia vida prolongada estende-se de um a quatro dias para a fluoxetina e de sete a 15 dias para seu metabólito ativo, a norfluoxetina. A excreção ocorre via renal, sendo menos de $10 \%$ eliminada na urina, sem sofrer alterações (Sena, 2009). Os efeitos colaterais mais relatados são gastrintestinais (náuseas, vômitos, dor abdominal, diarreia), psiquiátricos (agitação, ansiedade, insônia, nervosismo), alterações no sono e no peso, reações dermatológicas e disfunções sexuais (Moreno et al., 2002).

Ramanathan, Kumar e Suresh (2003) dizem que a fluoxetina pode melhorar as condições cognitivas de deprimidos. Essa melhora se daria principalmente na capacidade de aprendizagem e na memória. Amado-Bocara, Gougolis, Poirier-Littré, Galinowski e Lôo (1994) indicam que a fluoxetina pode aumentar os escores em testes cognitivos em pacientes deprimidos e em sujeitos normais. Cassano, Puca, Scapicchio e Trabucchi (2002) apontam que a fluoxetina ajuda a melhorar a memória e a atenção, além do humor dos pacientes. Por outro lado, alguns estudos (Mirow, 1991) associam o uso de fluoxetina com disfunção cognitiva, e outros (Dunkin et al., 2000) dizem que problemas na área executiva da cognição podem prever uma resposta precária dos pacientes medicados com fluoxetina.

Alguns pesquisadores questionam em qual tipo de depressão os medicamentos devem ser utilizados em conjunto com o tratamento psicoterápico (De Jonghe \& et al., 2004; De Jonghe, Kool, Van Aalst, Dekker, \& Peen, 2001; Greenberg \& Goldman, 2009). Os tratamentos combinados (psicoterapia e medicamento) têm sido utilizados principalmente em depressões graves, com risco de vida, e/ou refratárias a um dos tratamentos (Tomba \& Fava, 2008).

Tendo em vista o exposto e conforme citado anteriormente, este trabalho tem o objetivo de analisar e comparar mudanças cognitivas em pacientes deprimidos ao longo de dois diferentes modelos de tratamento, a saber: tratamento em psicoterapia psicodinâmica e tratamento biológico com fluoxetina, durante o período de 12 meses.

\section{Método}

\section{Participantes}

Os critérios de inclusão para participar do estudo foram: presença de transtorno depressivo maior ou transtorno depressivo não especificado de outra forma, de acordo com os critérios do DSM-IV-TR e sintomas depressivos moderados (BDI entre 20-35). Os critérios de exclusão, por sua vez, foram: risco de suicídio, uso de outros medicamentos que pudessem influenciar o funcionamento mental, doenças somáticas graves, história de problemas neurológicos e contraindicação para o tratamento com fluoxetina.

A amostra foi constituída por 180 adultos diagnosticados com Transtorno Depressivo Maior de nível moderado. Desses, 156 preencheram os critérios de inclusão e foram divididos em dois grupos de tratamento (psicoterapia ou fluoxetina). A idade dos participantes no grupo de psicoterapia $(n=80)$ variou entre 26 e 34 anos $(M=29,84 ; D P=2,42)$, sendo que $64,2 \%$ eram do sexo feminino. A maioria $(54,2 \%)$ possuía ensino superior e era solteira $(55,4 \%)$. No grupo de fluoxetina $(n=76)$, a idade variou também variou entre 26 e 34 
$\operatorname{anos}(\mathrm{M}=29,52 ; \mathrm{DP}=2,25)$, e 73,9\% eram mulheres. Nesse grupo, $49,3 \%$ tinham curso superior e $52,2 \%$ eram solteiros.

\section{Instrumentos}

Neste estudo, o instrumento principal foi a Escala de Inteligência Wechsler para Adultos, Terceira Edição (WAIS-III). Para entrada no estudo, entretanto, os participantes responderam ainda a um Questionário de Dados Sociodemográficos, e ao Inventário de Depressão de Beck (BDI).

Questionário de Dados Sociodemográficos: Esse questionário contemplou questões como sexo, idade, nível sociocultural, entre outras questões consideradas relevantes para o estudo.

Inventário de Depressão de Beck (BDI): O Inventário de Depressão de Beck (Beck \& Steer, 1993) é provavelmente a medida de autoavaliação de intensidade de depressão mais amplamente utilizada tanto em pesquisa como na clínica (Dunn, Sham, \& Hand, 1993), tendo sido traduzida para vários idiomas e validada em diferentes países. A versão utilizada para o presente estudo foi adaptada e validada por Cunha (2001). A escala consiste de 21 itens, incluindo sintomas e atitudes, cuja intensidade varia de 0 a 3 . Os itens referem-se a tristeza, pessimismo, sensação de fracasso, falta de satisfação, sensação de culpa, sensação de punição, autodepreciação, autoacusações, ideias suicidas, crises de choro, irritabilidade, retração social, indecisão, distorção da imagem corporal, inibição para o trabalho, distúrbio do sono, fadiga, perda de apetite, perda de peso, preocupação somática e diminuição de libido. Ao final do levantamento, quando os escores são somados, pode-se fazer a classificação dos sintomas depressivos de intensidade mínima (0-11 pontos), leve (12-19 pontos), moderada (20 a 35 pontos) e grave (36-63 pontos).

Escala de Inteligência Wechsler para Adultos, Terceira Edição (WAIS-III): A Escala de Inteligência Wechsler para Adultos (do inglês, Wechsler Adult Intelligence Scale WAIS-III), $3^{\text {a }}$ edição, é um dos mais importantes testes para avaliação clínica de capacidade intelectual de adultos na faixa etária entre 16 e 89 anos. É um instrumento de medida do funcionamento intelectual e cognitivo da pessoa sob uma forma abrangente e global (Cunha, 2000; Nascimento, 2004). Esse instrumento fornece subsídios para identificar e avaliar o estado e funcionamento das condições intelectuais e da cognição como um todo. A WAIS-III foi adaptada à população brasileira (Nascimento, 2004), e é amplamente utilizada por tratar-se de uma bateria fechada, com 14 subtestes que avaliam diversas funções cognitivas (Lezak, Howieson, \& Loring, 2004). Os subtestes são: Vocabulário, Semelhanças, Aritmética, Dígitos, Informação, Compreensão, Sequência de Número e Letras, Completar Figuras, Códigos, Cubos, Raciocínio Matricial, Arranjo de Figuras, Procurar Símbolos e Armar Objetos. Um estudo de confiabilidade teste-reteste da WAIS-III foi realizado com uma subamostra do presente estudo (Bastos, Gomes, Bandeira, \& Trentini, 2011), a fim de garantir a confiabilidade da WAIS-III em pacientes deprimidos. Os coeficientes de confiabilidade encontrados ficaram entre 0,72 e 0,99, e se mostraram adequados para o presente estudo.

\section{Procedimento}

Os participantes foram selecionados entre os pacientes que buscaram atendimento em um ambulatório de saúde mental da região metropolitana de Porto Alegre/Brasil. Eles foram inicialmente entrevistados por um psicólogo clínico. Pacientes com sinais de transtorno depressivo e ausência de critérios claros de exclusão foram convidados para uma avaliação inicial uma semana depois.

Os critérios de inclusão e exclusão foram verificados novamente com a entrevista clínica estruturada para o DSM, SCID-I e SCID-II (Del-Ben et al., 2001; Del-Ben, Rodrigues, \& Zuardi, 1996; Del-Ben, Zuardi \& Rodrigues, 1998), e pelo BDI, a fim de quantificar a gravidade do episódio depressivo. Após a confirmação do diagnóstico, a pesquisa e seus objetivos foram explicados. Pesquisadores independentes avaliaram os pacientes com a WAIS-III antes e depois dos tratamentos. Esses pesquisadores não sabiam que tratamentos os pacientes recebiam. O estudo seguiu as recomendações da Declaração de Helsinque, e o consentimento informado dos participantes foi obtido. O projeto do estudo foi aprovado pelo Comitê de Ética local.

A psicoterapia psicodinâmica utilizada no presente estudo foi similar ao modelo proposto por Gabbard (2004, 2010). Esse modelo de psicoterapia funciona dentro de um contexto interpretativo e elucidativo, no qual é enfatizada a construção de vínculo positivo com o paciente. $\mathrm{O}$ modelo conceitual desse tratamento inclui a compreensão dos conflitos inconscientes do paciente, e deriva das teorias psicanalíticas (como a psicologia do ego, teoria das relações de objeto, a psicologia do self, a teoria do apego e a psicanálise clássica). A psicoterapia foi realizada individualmente, em consultas semanais.

Para os pacientes expostos ao tratamento biológico, a fluoxetina, foi prescrita por médicos psiquiatras, de acordo com o protocolo proposto pelo Ministério da Saúde e Agência Nacional de Vigilância Sanitária (ANVISA). Essas recomendações começam com $20 \mathrm{mg}$ de fluoxetina por dia durante duas a quatro semanas e, se necessário, aumento gradual da dose até um máximo de $60 \mathrm{mg}$ por dia (http://www4.anvisa. gov.br/base/visadoc/BM/BM(34652-1-0).PDF). A fluoxetina foi escolhida pela sua excelente relação custo-benefício em comparação com os custos de tratamento da depressão (Salminen et al., 2008).

Na primeira consulta psiquiátrica, os pacientes receberam explicações sobre o tratamento medicamentoso e receberam fluoxetina suficiente para duas semanas de tratamento, quando, então, tiveram a segunda consulta. As consultas quinzenais continuaram até que a dose adequada da medicação fosse atingida. Depois disso, os pacientes passaram a comparecer a consultas mensais, nas quais a adesão ao tratamento era verificada, e eles recebiam o medicamento.

\section{Análise de dados}

Os dados foram analisados a partir de estatísticas descritivas e inferenciais. Inicialmente, para a estatística descritiva foram utilizadas frequência, média e desvio-padrão. A estatística inferencial utilizada foi o Teste $t$. Além disso, foi utilizada a MANOVA de medidas repetidas, a fim de 
também realizar procedimentos multivariados para avaliação de diferenças de grupos. A variância das médias obtidas foi verificada, considerando-se a variável "grupo de tratamento" (Aron, Aron, \& Coups, 2006; Hair, Black, Babin, Anderson, \& Tatham, 2005). Tais análises foram efetuadas utilizando o software Statistical Package for the Social Sciences (SPSS), versão 18 .

\section{Resultados e Discussão}

Em um primeiro momento, antes da apresentação dos resultados referentes à influência exercida pelos tratamentos sobre a cognição do pacientes, cabe ressaltar que os pressupostos exigidos pelas análises estatísticas inferenciais foram atendidos. Esses pressupostos incluem normalidade multivariada, homogeneidade da variância, linearidade e multicolinealidade (Hair et al., 2005).
Os resultados de Teste $t$ dentro de cada grupo mostraram uma melhora significativa para nove dos 14 subtestes - cerca de $64 \%$, para os pacientes em psicoterapia. Os pacientes em terapia biológica, por sua vez, obtiveram uma melhora significativa em quatro dos 14 subtestes avaliados - cerca de $28 \%$. Esses resultados podem ser observados na Tabela 1.

$\mathrm{Na}$ comparação teste-reteste, o grupo de psicoterapia melhorou nas áreas de Inteligência Verbal e de Execução. Os pacientes melhoraram significativamente $(p<0,05)$ nas funções cognitivas de memória de trabalho (Dígitos e Sequência de Números e Letras), atenção e velocidade de processamento (Códigos e Procurar Símbolos), raciocínio abstrato (Raciocínio Matricial), capacidade de julgamento e senso comum (Compreensão e Arranjo de Figuras), capacidade de percepção do óbvio, capacidade de organização, maleabilidade associativa e capacidade para enfrentar uma situação (Arranjo de Figuras).

Tabela 1. Resultados de média, desvio-padrão, teste $t$ e nível de significância dos dois grupos de tratamento.

\begin{tabular}{|c|c|c|c|c|}
\hline Subteste & $\begin{array}{c}\text { Teste } \\
\text { Média }(D P)\end{array}$ & $\begin{array}{c}\text { Reteste } \\
\text { Média }(D P)\end{array}$ & $t$ & $p$ \\
\hline \multicolumn{5}{|l|}{ Grupo Psicoterapia $(\mathrm{n}=80)$} \\
\hline Vocabulário & $13,03(3,47)$ & $13,06(3,23)$ & 0,53 & 0,59 \\
\hline Semelhanças & $13,48(3,16)$ & $13,51(2,84)$ & $-0,44$ & 0,65 \\
\hline Aritmética & $11,97(3,35)$ & $12,15(2,50)$ & $-2,03$ & 0,06 \\
\hline Dígitos & $9,90(2,36)$ & $11,17(1,74)$ & $-3,10$ & $0,01^{*}$ \\
\hline Informação & $11,89(3,09)$ & $12,09(2,83)$ & $-1,07$ & 0,28 \\
\hline Compreensão & $12,69(3,47)$ & $13,03(3,07)$ & $-1,13$ & $0,02 *$ \\
\hline Seqüência de Números e Letras & $9,73(2,28)$ & $10,80(1,63)$ & $-2,16$ & $0,03 *$ \\
\hline Completar Figuras & $12,13(2,57)$ & $12,22(1,88)$ & $-0,89$ & 0,37 \\
\hline Códigos & $10,25(2,31)$ & $11,97(1,67)$ & $-3,96$ & $0,01^{*}$ \\
\hline Cubos & $12,70(2,76)$ & $13,20(2,26)$ & $-2,62$ & $0,01^{*}$ \\
\hline Raciocínio Matricial & $11,14(2,11)$ & $12,01(1,60)$ & $-3,57$ & $0,01^{*}$ \\
\hline Arranjo de Figuras & $10,94(2,72)$ & $11,31(2,24)$ & $-2,37$ & $0,02 *$ \\
\hline Procurar Símbolos & $11,30(2,56)$ & $11,68(1,91)$ & $-2,47$ & $0,01 *$ \\
\hline Armar Objetos & $11,12(2,67)$ & $11,96(2,48)$ & $-2,03$ & $0,04 *$ \\
\hline \multicolumn{5}{|l|}{ Grupo Fluoxetina $(\mathrm{n}=76)$} \\
\hline Vocabulário & $12,85(3,27)$ & $12,81(3,20)$ & 0,19 & 0,84 \\
\hline Semelhanças & $13,19(2,64)$ & $13,20(2,56)$ & $-1,00$ & 0,32 \\
\hline Aritmética & $11,86(3,07)$ & $11,95(2,89)$ & $-2,05$ & $0,04 *$ \\
\hline Dígitos & $9,95(2,21)$ & $10,15(2,03)$ & $-2,41$ & $0,01 *$ \\
\hline Informação & $11,88(2,84)$ & $11,93(2,71)$ & $-1,93$ & 0,06 \\
\hline Compreensão & $12,80(3,39)$ & $12,87(3,21)$ & $-0,68$ & 0,49 \\
\hline Seqüência de Números e Letras & $9,85(2,26)$ & $9,92(2,22)$ & $-1,42$ & 0,15 \\
\hline Completar Figuras & $12,20(2,39)$ & $12,25(2,27)$ & $-0,89$ & 0,37 \\
\hline Códigos & $10,36(2,44)$ & $10,70(2,03)$ & $-3,62$ & $0,01^{*}$ \\
\hline Cubos & $12,62(2,85)$ & $12,87(2,66)$ & $-2,50$ & $0,01^{*}$ \\
\hline Raciocínio Matricial & $11,05(2,08)$ & $11,39(1,91)$ & $-3,35$ & 0,10 \\
\hline Arranjo de Figuras & $10,85(2,72)$ & $10,88(2,68)$ & $-2,95$ & 0,40 \\
\hline Procurar Símbolos & $11,32(2,59)$ & $11,49(2,30)$ & $-1,93$ & 0,06 \\
\hline Armar Objetos & $11,12(2,71)$ & $11,21(2,53)$ & $-1,84$ & 0,07 \\
\hline
\end{tabular}

Nota: *Diferença estatisticamente significativa $(\mathrm{p}<0,05)$. 
Figura 1. Comparação das médias dos subtestes com alteração estatisticamente significativa no pré-tratamento e no pós-tratamento nos dois grupos.

Efeitos da Psicoterapia vs Efeitos da Fluoxetina

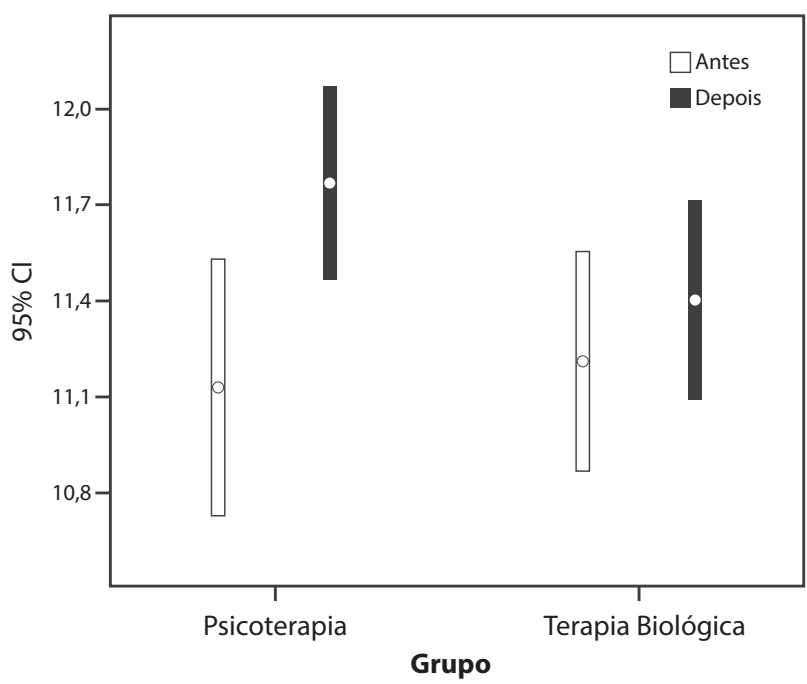

O grupo de terapia biológica melhorou especialmente na área da Inteligência de Execução. As principais melhoras parecem associadas às funções de memória de trabalho (Dígitos e Sequência de Números e Letras), raciocínio abstrato (Raciocínio Matricial), atenção, concentração, velocidade de processamento cognitivo (Procurar Símbolos e Códigos) e raciocínio espacial (Cubos). Estudos têm relatado a capacidade da fluoxetina de induzir neurogênese no hipocampo, com consequente melhora cognitiva nas funções de memória (Li et al., 2009; Mowla, Mosavinasab, \& Pani, 2007), o que parece sustentar os presentes achados. Teng, Humes, Nakata e Melzer (2008) e Ogren et al. (2008) também já haviam apontado que a estimulação do sistema serotoninérgico aparentemente está relacionada à melhora da cognição.

A Figura 1 demonstra as diferenças de mudança nos escores médios dos subtestes com alterações estatisticamente significativas. O grupo de psicoterapia psicodinâmica obteve 11,1 pontos ponderados, em média, no pré-tratamento, subindo, após 12 meses de intervenção, para 11,9 pontos. $\mathrm{O}$ grupo de fluoxetina, por sua vez, obteve no pré-tratamento a média de 11,2 pontos, e no pós-tratamento, a média ficou em 11,4 pontos ponderados.

Os resultados sugerem que tanto a psicoterapia psicodinâmica quanto a terapia biológica com fluoxetina possibilitam boa resposta de melhora cognitiva em pacientes deprimidos com nível até moderado de intensidade dos sintomas depressivos. Essa melhora aparentemente já é mensurável cognitivamente 12 meses após o início dos tratamentos em alguns subtestes da WAIS-III.

A psicoterapia psicodinâmica mostrou melhora cognitiva em pacientes deprimidos não medicados. Essa resposta parece demonstrar um efeito bastante amplo da psicoterapia psicodinâmica sobre a cognição, atingindo tanto o aspecto verbal, como o de execução da inteligência. A terapia biológica com fluoxetina, por sua vez, aparentemente também possibilita boa resposta de melhora cognitiva. Algumas funções cognitivas, como atenção, memória de trabalho e velocidade de processamento, parecem ser especialmente sensíveis a intervenção biológica, que demonstra ser mais efetiva no aspecto de execução do que no aspecto verbal da inteligência.

Para verificar a variância das médias obtidas nos subtestes foi realizada a MANOVA, envolvendo os dois grupos (psicoterapia psicodinâmica e fluoxetina), dois momentos de avaliação (antes e depois do tratamento) e 14 subtestes da WAIS-III (variáveis dependentes). Os resultados dessa análise são apresentados na Tabela 2 .

De acordo com os dados da Tabela 2, nota-se que há uma diferença significativa entre os grupos nas pontuações médias obtidas na segunda avaliação (as médias da avaliação inicial não tiveram diferença significativa). Essas diferenças envolvem os subtestes Vocabulário, Aritmética, Dígitos, Compreensão, Sequência de Números e Letras, Completar Figuras e Códigos. Aparentemente, as variáveis cognitivas avaliadas por esses instrumentos sofrem

Tabela 2. Resultados da análise de variância investigando o efeito grupo de tratamento.

\begin{tabular}{clcc}
\hline Variáveis Independentes & Variável Dependente & $F$ & $p$ \\
\hline Psicoterapia Psicodinâmica & Vocabulário & 4,46 & $0,03^{*}$ \\
\cline { 1 - 2 } Fluoxetina & Semelhanças & 1,20 & 0,27 \\
& Aritmética & 12,87 & $0,01^{*}$ \\
& Dígitos & 11,27 & $0,01^{*}$ \\
& Informação & 3,75 & 0,06 \\
& Compreensão & 6,31 & $0,01^{*}$ \\
& Sequência de Números e Letras & 22,46 & $0,01^{*}$ \\
& Completar Figuras & 13,42 & $0,01^{*}$ \\
& Códigos & 4,31 & $0,03^{*}$ \\
& Cubos & 0,11 & 0,73 \\
& Raciocínio Matricial & 0,10 & 0,74 \\
& Arranjo de Figuras & 0,42 & 0,51 \\
& Procurar Símbolos & 1,23 & 0,26 \\
& Armar Objetos & 0,15 & 0,69 \\
\hline
\end{tabular}

Nota: * Diferença estatisticamente significativa $(\mathrm{p}<0,05)$. 
influências mais acentuadas por efeito da psicoterapia psicodinâmica do que pelo efeito da terapia biológica, em pacientes deprimidos.

Os resultados encontrados no grupo de psicoterapia aproximam-se do que foi encontrado por Yázigi et al. (2011). Esses pesquisadores avaliaram 34 pacientes com a WAIS-III antes de iniciar psicoterapia psicanalítica, e os reavaliaram um e dois anos após a avaliação basal. Os resultados mostraram um aumento na capacidade de atenção (Completar Figuras) e na velocidade de processamento cognitivo (Procurar Símbolos) nos pacientes deprimidos avaliados. O subteste Arranjo de Figuras também mostrou diferenças significativas na segunda avaliação. Um aspecto importante sobre o subteste Arranjo de Figuras pode ser ressaltado. Seus resultados têm sido associados à capacidade de planejar, antecipar e interpretar situações sociais, e à capacidade de antecipar as consequências dos comportamentos das pessoas (Lezak et al., 2004). Yázigi et al. (2011) interpretaram o significado dos resultados do Arranjo de Figuras como "significando uma elevação do pensamento social adequado, a fim de discriminar entre percepções relevantes e não relevantes, que possibilitam melhores respostas sociais" (p. 17).

Os subtestes em que foram observadas melhoras relacionadas à psicoterapia psicodinâmica (no caso, Compreensão e Arranjo de Figuras) apontam, assim, para uma ampliação da maturidade social, do senso comum, da aptidão crítica em relação à própria experiência e um maior ajustamento à realidade. Essa "elevação" dos processos cognitivos relacionados à interação social é parte constituinte da dinâmica interacional trabalhada com os pacientes nas psicoterapias psicodinâmicas (para uma discussão mais profunda dos conceitos e princípios da psicoterapia psicodinâmica, consultar Gabbard, 2010; McWilliams, 2004).

Assim, é importante considerar que esses resultados podem sugerir uma sensibilidade psicométrica do Arranjo de Figuras da WAIS-III para detectar alterações cognitivas específicas em pacientes deprimidos que estão recebendo psicoterapia psicodinâmica. No entanto, deve ser feita a ressalva que essa consideração é meramente hipotética, pois as amostras de participantes dos dois estudos são muito diferentes, tanto em tamanho amostral, como em perfis sociodemográficos e clínicos. No estudo de Yázigi et al. (2011), os participantes formaram um grupo heterogêneo de pacientes com diagnósticos variados, enquanto que no presente estudo os participantes formaram uma amostra muito homogênea. Novas investigações poderiam esclarecer essas considerações específicas.

Como dito anteriormente, Dunkin et al. (2000) já haviam sugerido que uma melhora cognitiva ocorre antes da melhora clínica da depressão. Dessa forma, pode-se pensar que a melhora cognitiva ampla proporcionada pela psicoterapia psicodinâmica pode servir como indício de melhora clínica. De qualquer modo, é importante o debate sobre o quanto essa melhora, embora significativa, impacta a vida dos pacientes, já que a diferença, na maioria dos subtestes, não foi classificatória e sim, matemática. Ou seja, a mudança cognitiva não foi suficiente para que o nível de classificação da habilidade mensurada fosse alterado.

\section{Considerações finais}

Os resultados obtidos neste estudo permitem afirmar que a psicoterapia psicodinâmica e a terapia biológica com fluoxetina têm efeito sobre a cognição de pacientes adultos deprimidos. Além disso, a psicoterapia psicodinâmica se mostrou mais eficaz em algumas das funções cognitivas variadas.

Cabe ressaltar, entretanto, que vários fatores podem influenciar na cognição dos pacientes. Esses fatores incluem as experiências cotidianas dos sujeitos, as alterações fisiológicas (mudanças hormonais, por exemplo), as alterações psicológicas (defesas, padrões comportamentais) e fatores culturais (valores, regras sociais, etc.).

De qualquer forma, a avaliação cognitiva de pacientes deprimidos parece oferecer a oportunidade de se conhecer as possíveis áreas da cognição que se encontram prejudicadas. Assim, permitem proceder na busca de tratamentos mais eficientes e mais de acordo com as necessidades dos pacientes. A partir de avaliações bem executadas, seria possível traçar uma forma de intervenção terapêutica voltada para os diferentes déficits que aparecem nos quadros depressivos, sejam eles cognitivos, fisiológicos ou sociais.

Nesse sentido, a psicoterapia psicodinâmica mostrou-se eficiente. Corroborando indícios dados por Kandel (2003), pode-se dizer que há razões para se acreditar que essa forma de tratamento, se bem conduzida, pode oferecer um novo referencial intelectual para o futuro da psiquiatria e da psicoterapia de orientação psicanalítica.

No caso da depressão, é de interesse saber sobre qual sistema neurotransmissor a técnica psicoterápica age. Como verificado no presente trabalho, houve melhora em funções cognitivas associadas a circuitos neurocognitivos variados, incluindo o córtex pré-frontal, o hipocampo e o circuito límbico-fronto-estriatal. Alguns estudos já haviam relacionado essas áreas ao humor deprimido (Frey et al., 2007; Porter et al., 2007).

No entanto, outras questões seguem sem resposta. Seriam os déficits cognitivos encontrados uma consequência da depressão? Ou seria o humor depressivo efeito da redução de funções cognitivas importantes para o funcionamento saudável do psiquismo? Novos estudos são sugeridos, já que essas respostas têm implicações terapêuticas importantes.

Enfim, tem-se melhora cognitiva em ambas as modalidades de tratamento. Entretanto, as evidências de melhora parecem mais marcadas e foram significativamente diferentes e favoráveis para o grupo de pacientes em psicoterapia psicodinâmica. A hipótese que fica, entre outras já destacadas no texto, é a de que a psicoterapia abriria um espaço para a discussão de estratégias e caminhos para a solução de possíveis déficits cognitivos. Esse estudo ainda está em andamento e outra modalidade de tratamento será incluída em outros trabalhos, nomeadamente o tratamento combinado de psicoterapia psicodinâmica e tratamento biológico, para futuras análises.

Conforme dizem Solms e Turbull (2002), se uma massa crítica de pesquisadores puder escolher o caminho de colocar suas terapêuticas à prova, o que vai ser conquistado será muito maior do que o esforço que se terá que fazer. Também será possível compreender melhor como distúrbios e síndromes neuropsiquiátricas se desenvolvem e se estruturam. 
Isso permitirá que nossas terapêuticas sejam direcionadas àqueles que se beneficiam verdadeiramente delas e de forma que funcionem melhor.

\section{Referências}

Amado-Bocara, I., Gougolis, N., Poirier-Litré, M., Galinowski, A., \& Lôo, H. (1994). Effects of antidepressants on cognitive functions. Review of the literature. Encephale, 20(1), 65-77.

American Psychiatry Association -APA. (2002). DSM-IV-Manual diagnóstico e estatístico de transtornos mentais. Porto Alegre: Artmed.

Aron, A., Aron, E., \& Coups, E. (2006). Statistics for psychology. New York: Pearson.

Bachrach, H., Galatzer-Levy, R., Skolnikoff, A., \& Waldron, S. (1991). On the efficacy of psychoanalysis. Journal of the American Psychoanalytic Association, 39(4), 871-916.

Bastos, A. G., Gomes, B. M., Bandeira, D. R., \& Trentini, C. M. (2011). Reliability of the Brazilian WAIS-III in depression. Interamerican Journal of Psychology, 45(3), 419-428.

Beck, A., \& Steer, R. (1993). Beck Depression Inventory. San Antonio, TX: Psychological Corporation.

Cassano, G. B., Puca, F., Scapicchio, P. L., \& Trabucchi, M. (2002). Paroxetine and fluoxetine effects on mood and cognitive functions in depressed nondemented elderly patients. The Journal of Clinical Psychiatry, 63, 396-402.

Chepenik, L., Cornew, L., \& Farah, M. (2007). The influence of sad mood on cognition. Emotion, 7(4), 802-811.

Cuijpers, P., Clignet, F., van Meijel, B., van Straten, A., Li, J., \& Andersson, G. (2011). Psychological treatment of depression in inpatients: A systematic review and meta-analysis. Clinical Psychology Review, 31, 353-360.

Cunha, J. (2000). Psicodiagnóstico- $V$. Porto Alegre: Artmed.

Cunha, J. (2001). Manual da versão em português das Escalas Beck. São Paulo: Casa do Psicólogo.

De Jonghe, F., Hendricksen, M., Van Aalst, G., Kool, S., Peen, V., Van, R., Van Den Eijnden, E., Dekker, J. (2004). Psychotherapy alone and combined with pharmacotherapy in the treatment of depression. British Journal of Psychiatry, 185, 37-45.

De Jonghe, F., Kool, S., Van Aalst, G., Dekker, J., \& Peen, V. (2001). Combining psychotherapy and antidepressants in the treatment of depression. Journal of Affective Disorders, 64, 217-229.

Del-Ben, C., Rodrigues, C., \& Zuardi, A. (1996). Reliability of the Portuguese version of the structured clinical interview for DSM-III-R (SCD-I) in a Brazilian sample of psychiatric outpatients. Brazilian Journal of Medical Research, 29(12), 1675-1682.

Del-Ben, C., Vilela, J., Crippa, J., Hallak, J., Labate, C., \& Zuardi, A. (2001). Confiabilidade teste-reteste da Entrevista Clínica Estruturada para o DSM-IV - Versão clínica traduzida para o português. Revista da Associação Brasileira de Psiquiatria, 23, 156-159.

Del-Ben, C., Zuardi, A., \& Rodrigues, C. (1998). Confiabilidade do diagnóstico psiquiátrico levantado sob supervisão e do diagnóstico obtido através da entrevista clínica estruturada para o DSM-III-R (SCID). Revista da Associação Brasileira de Psiquiatria, 20, 140-145.
Dobson, K. (1989). A meta-analysis of the efficacy of cognitive therapy for depression. Journal of Consulting and Clinical Psychology, 57, 414-419.

Drevets, W. (2003). Neuroimaging abnormalities in the amygdala in mood disorders. Annals of the New York Academy of Sciences, 985, 420-444.

Dunkin, J., Leuchter, J., Cook, I., Kasl-Godley, J., Abrams, M., \& Rosenberg-Thompson, S. (2000). Executive dysfunction predicts non-response to fluoxetine in major depression. Journal of Affective Disorders, 60, 13-23.

Dunn, G., Sham, P., \& Hand, D. (1993). Statistics and nature of depression. Psychological Medicine, 23, 871-889.

Eugene, F., Levesque, J., Mensour, B., Leroux, J., Beaudoin, G., $\&$ Bourgouin, P. (2003). The impact of individual differences on the neural circuitry underlying sadness. NeuroImage, 19, 354-364.

Fonagy, P., Roth, A., \& Higgitt, A. (2005). Psychodynamic therapies, evidence-based practice and clinical wisdom. Bulletin of the Menninger Clinic, 69(1), 01-58.

Freud, S. (1996). Sobre a psicoterapia (J. Salomão, Trad.). Em J. Strachey (Ed.), Obras psicológicas completas de Sigmund Freud: edição standard brasileira (Vol. VII, pp. 241-254). Rio de Janeiro: Imago. (Trabalho original publicado em 1905)

Freud, S. (1996). Linhas de progresso na terapia psicanalítica (J. Salomão, Trad.). Em J. Strachey (Ed.), Obras psicológicas completas de Sigmund Freud: edição standard brasileira (Vol. XVII, pp. 169-181). Rio de Janeiro: Imago. (Trabalho original publicado em 1918)

Freud, S. (1996). Novas conferências introdutórias sobre psicanálise (J. Salomão, Trad.). Em J. Strachey (Ed.), Obras psicológicas completas de Sigmund Freud: edição standard brasileira (Vol. XXII, pp. 11-178). Rio de Janeiro: Imago. (Trabalho original publicado em 1933)

Freud, S. (1996). Construções em análise (J. Salomão, Trad.). Em J. Strachey (Ed.), Obras psicológicas completas de Sigmund Freud: edição standard brasileira (Vol. XXIII, pp. 271-288). Rio de Janeiro: Imago. (Trabalho original publicado em 1937)

Frey, B., Andreazza, A., Nery, F., Martins, M., Quevedo, J., Soares, J., Kapczinski, F. (2007). The role of hippocampus in the pathophysiology of bipolar disorder. Behavioral Pharmacology, 18 (5-6), 419-430.

Gabbard, G. (2000). A neurobiologically informed perspective on psychotherapy. British Journal of Psychiatry, 177, 117-122.

Gabbard, G. (2004). Long-term psychodynamic psychotherapy: A basic text. Arlington, TX: American Psychiatric Publishing.

Gabbard, G. (2008). Textbook of psychotherapeutic treatments in psychiatry. Washington, DC: American Psychiatric Publishing.

Gabbard, G. (2010). Long-term psychodynamic psychotherapy: A basic text (2nd ed.). Arlington, TX: American Psychiatric Publishing.

Greenberg, R., \& Goldman, E. (2009). Antidepressants, psychotherapy or their combination: Weighing options for depression treatments. Journal of Contemporary Psychotherapy, 39, 83-91.

Greene, L. (1993). Primitive defenses and the borderline patient's perceptions of the psychiatric treatment team. Psychoanalytic Psychology, 10(4), 533-549.

Hair, J., Black, W., Babin, B., Anderson, R., \& Tatham, R. (2005). Multivariate data analysis. New Jersey: Prentice Hall. 
Harvey, P., Le Bastard, G., Pochon, J., Levy, R., Allilaire, J., \& Dubois, B. (2004). Executive functions and updating of the contents of working memory in unipolar depression. Journal of Psychiatric Research, 38, 567-576.

Hyman, S. (1999). Looking to the future: The role of genetics and molecular biology in research on mental illness, in psychiatry in the new millenium. Washington, DC: American Psychiatric Publishing.

Kandel, E. (2003). A biologia e o futuro da psicoterapia psicanalítica: um novo referencial intelectual para a psiquiatria revisitado. Revista de Psiquiatria do Rio Grande do Sul, 25(1), 139-165.

Kaplan, B., \& Sadock, V. (2008). Kaplan \& Sadock's concise textbook of psychiatry. Philadelphia: Lippincott, Willians \& Wilkins.

Kaplan-Solms, K., \& Solms, M. (2005). Estudos clínicos em neuropsicanálise: introdução a uma neuropsicologia profunda. São Paulo: Lemos.

Lamb, W. (2005). A meta-analysis of outcome studies in long-term psychodynamic psychotherapy and psychoanalysis. Berkeley, CA: University of California.

Lambert, M., \& Ogles, B. (2004). The efficacy and effectiveness of psychotherapy. In M. Lambert (Ed.), Bergin and Garfield's handbook of psychotherapy and behavior change (pp. 139193). New York: John Wiley \& Sons.

Leichsenring, F., \& Rabung, S. (2008). Effectiveness of long-term psychodynamic psychotherapy: A meta-analysis. JAMA, 3000(13), 1551-1565.

Leuzinger-Bohleber, M., Stuhr, U., Bernhard, R., \& Beutel, M. (2003). How to study the 'quality of psychoanalytic treatments' and their long-term effects on patients' wellbeing: a representative, multi-perspective follow-up study. The International Journal of Psychoanalysis, 84(2), 263-290.

Lezak, M., Howieson, D., \& Loring, D. (2004). Neuropsychological assessment (4th ed.) Oxford, UK: University Press.

Li, W., Cai, H., Wang, B., Chen, L., Zhou, Q., Luo, C., Liu, N., Ding, X., Zhu, D. (2009). Chronic Fluoxetine treatment improves ischemia-induced spatial cognitive deficits through increasing hippocampal neurogenesis after stroke. Journal of Neuroscience Research, 87(1), 112-122.

Machado, S., \& Vasconcellos, M. (1998). Psicanálise e psicoterapia de orientação analítica. In A. Cordioli (Ed.), Psicoterapias: abordagens atuais (pp. 137-144). Porto Alegre: Artmed.

Marvel, C., \& Paradiso, S. (2004). Cognitive and neurological impairments in mood disorders. Psychiatric Clinics of North America, 27, 19-36.

McWilliams, N. (2004). Psychoanalytic psychotherapy: A practioner's guide. New York: Guilford.

Miller, W. (1975). Psychological deficit in depression. Psychological Bulletin, 82(2), 238-260.

Mirow, S. (1991). Cognitive dysfunction associated with fluoxetine. The American Journal of Psychiatry, 148(7), 948-949.

Moreno, R., Moreno, D., \& Soares, M. (2002). Psicofarmacologia de antidepressivos. In J. Mari, G. Busatto Filho, I. Bordin, \& E. Miguel Filho (Eds.), Atualização em psiquiatria I (pp. 51-75). São Paulo: Casa do Psicólogo.

Mowla, A., Mosavinasab, M., \& Pani, A. (2007). Does fluoxetine have any effect on the cognition of patients with mild cognitive impairment? A double-blind, placebo-controlled, clinical trial. Journal of Clinical Psychopharmacology, 27(1), 67-70.
Murphy, F., Nimmo-Smith, I., \& Lawrence, A. (2003). Functional neuroanatomy of emotions: A meta-analysis. Cognitive, Affective, and Behavioral Neuroscience, 3, 207-233.

Nascimento, E. (2004). Manual da Escala de Inteligência Wechsler para adultos. São Paulo: Casa do Psicólogo.

Ogren, S., Eriksson, T., Elvander-Tottie, E., D’Addario, C., Ekström, J., Svenningsson, P., Meister, B., Kehr, J., Stiedl, O. (2008). The role of 5-HT(1A) receptors in learning and memory. Behavioral Brain Research, 16, 195(1), 54-77.

Philips, M., Drevets, W., Rauch, S., \& Lane, R. (2003a). Neurobiology of emotional perception I: The neural basis of normal emotion perception. Biological Psychiatry, 54, 504-514.

Philips, M., Drevets, W., Rauch, S., \& Lane, R. (2003b). Neurobiology of emotional perception II: Implications for major psychiatric disorders. Biological Psychiatry, 54, 515528.

Porter, R., Bourke, C., \& Gallagher, P. (2007). Neuropsychological impairment in major depression: Its nature, origin and clinical significance. Australian and Neo Zealander Journal of Psychiatry, 41, 115-126.

Ramanathan, M., Kumar, S., \& Suresh, B. (2003). Evaluation of cognitive function of fluoxetine, sertraline and tianeptine in isolation and chronic unpredictable mild stress-induced depressive Wistar rats. Indian Journal of Experimental Biology, 41(11), 1269-1272.

Robbins, T., Joyce, E., \& Sahakian, B. (1992). Neuropsychology and neuroimaging. In E. Paykel (Ed.), Handbook of affective disorders (pp. 437-500). Edimburgh, UK: Churchill Livingstone.

Robinson, G. (2004). Genome mix: Beyond nature and nurture. Science, 304, 397-399.

Rogers, R., Kasai, K., Koji, M., Fukuda, R., Iwanami, A., \& Nakagome, K. (2004). Executive and prefrontal dysfunction in unipolar depression: A review of neuropsychological and imaging evidence. Neuroscience Research, 50, 1-11.

Roth, A., \& Fonagy, P. (2005). What works for whom? A critical review of psychotherapy research. New York: Guilford.

Salminen, J., Karlsson, H., Hietala, J., Kajander, J., Aalto, S., Markkula, J., Rasi-Hakala, H., Toikka, T. (2008). Shortterm psychodynamic psychotherapy and fluoxetine in major depressive disorder: A randomized comparative study. Psychotherapy and Psychossomatics, 77, 351-357.

Sena, E. (2009). Tratamento farmacológico da depressão. In A. Lacerda, L. Quarantini, A. Miranda-Scippa, \& J. Del Porto (Eds.), Depressão: do neurônio ao funcionamento social (pp. 269-286). Porto Alegre: Artmed.

Solms, M., \& Turnbull, O. (2002). The brain and the inner world: An introduction to the neuroscience of subjective experience. London: Karnac Books.

Steele, J., \& Lawrie, S. (2004). Segregation of cognitive and emotional function in the prefrontal cortex: Stereotactic metaanalysis. NeuroImage, 21, 868-875.

Teng, C., Humes, E., Nakata, A., \& Melzer, D. (2008). Modelos bioquímicos. In Moreno, R., \& Moreno, D. (Eds.), Da psicose maníaco-depressiva ao espectro bipolar (pp. 301-326). São Paulo: Editora Segmento Farma.

Tolman, A. (2009). Depressão em adultos: as mais recentes estratégias de avaliação e tratamento. Porto Alegre: Artmed. 
Tomba, E., \& Fava, G. (2009). The sequential combination of pharmacotherapy and psychotherapy in mood disorders. Journal of Contemporary Psychotherapy, 39, 101-109.

Vittengl, J., Clark, L., Dunn, T., \& Jarrett, R. (2007). Reducing relapse and recurrence in unipolar depression: A comparative analysis of cognitive-behavioral therapy's effects. Journal of Consulting and Clinical Psychology, 75, 475-488.

Wallerstein, R. (1986). Forty-two lives in treatment: A study of psychoanalysis and psychotherapy. New York: Guilford.

Westen, D., \& Morrison, K. (2001). A multidimensional metaanalysis of treatments for depression, panic, and generalized anxiety disorder: An empirical examination of the status of empirically supported therapies. Journal of Consulting and Clinical Psychology, 69, 875-899.

World Health Organization - WHO (2009). The world health report. Recuperado em 27 de novembro de 2012, de http://www.who. int/whr/2009/en/index.html
Yazigi, L., Botelho, N., Semer, N., Amaro, T., Fiore, M., \& Silva, J. (2011). Rorschach and the WAIS-III after one and two years of psychotherapy. Psicologia: Reflexão e Crítica, 24(1), 10-18.

Zorzetto Filho, D. (2009). Neuropsicologia da depressão. In A. Lacerda, L. Quarantini, A. Miranda-Scippa, \& J. Del Porto (Eds.), Depressão: do neurônio ao funcionamento social (pp. 253-268). Porto Alegre: Artmed.

Recebido em 19.10.2011

Primeira decisão editorial em 11.06.2013

Versão final em 14.06.2013

Aceito em 25.06.2013 